\title{
Biomarcadores histológicos em duas espécies de bagres estuarinos da Costa Maranhense, Brasil
}

[Histology biomarkers in two estuarine catfish species from the Maranhense Coast, Brazil]

\author{
D.B.P. Sousa ${ }^{1,2}$, Z.S. Almeida ${ }^{1}$, R.N.F. Carvalho-Neta ${ }^{1}$ \\ ${ }^{1}$ Universidade Estadual do Maranhão - São Luís, MA \\ ${ }^{2}$ Bolsista de Iniciação Científica do CNPq
}

\begin{abstract}
RESUMO
Neste estudo objetivou-se validar lesões branquiais como biomarcadores em peixes de importância econômica (Sciades herzbergii e Bagre bagre) capturados pela pesca artesanal em dois locais diferenciados da Baía de São Marcos, MA, a fim de selecionar um táxon como bioindicador capaz de ser utilizado em programas de biomonitoramento. O primeiro ponto (S1) foi utilizado como uma área de referência - Ilha dos Caranguejos -, e o segundo ponto (S2) foi considerado potencialmente impactado Complexo Portuário de São Luís, MA. Encontraram-se várias alterações histológicas para as duas espécies em S2, destacando-se: estreitamento lamelar, teleangectasia, fusão e descolamento do epitélio da lamela secundária. Lesões branquiais em $B$. bagre coletados em S1 também foram observadas, não sendo possível diferenciar os indivíduos das duas áreas analisadas. Os dados indicam que $S$. herzbergii é um bioindicador mais apropriado para análise de biomarcadores de contaminação aquática.
\end{abstract}

Palavras-chaves: bioindicador, contaminação aquática, monitoramento ambiental, peixe, Maranhão

\begin{abstract}
This study aimed to validate branchial lesions as a biomarker in fish (Sciades herzbergii and Bagre bagre) caught by traditional fishing in two different places at São Marcos Bay, MA, in order to select a bioindicator taxon which can be used in biomonitoring programs. The first point (S1) was used as a reference area - Caranguejos (Crab) Island and the second point (S2) was considered potentially impacted - Port Complex of São Luis, MA. Several histological changes were found for both species in S2, including: narrowing lamellar, teleangectasy, fusion and separation of secondary lamellar epithelium. Gill lesions in B. bagre collected were also observed in S1, it is not possible to differentiate between individuals of the two areas analyzed. The data indicate that $\mathrm{S}$. herzbergii is more appropriate as a bioindicator for analysis of biomarkers of aquatic contamination.
\end{abstract}

Keywords: bioindicator, aquatic contamination, Maranhão, environmental monitoring, fish

\section{INTRODUÇÃO}

Em um ambiente degradado, particularmente onde poluentes ocorrem em concentrações crônicas e subletais, mudanças na estrutura e função de organismos aquáticos são mais frequentes que mortalidade em massa. Nesse contexto, os peixes têm sido utilizados como um importante modelo biológico com o objetivo de investigar as potenciais interações químicas dos xenobióticos (Fernandes, 2005), especialmente com uso dos chamados biomarcadores. Segundo Huggett et al. (1992), os biomarcadores são comumente descritos como alterações bioquímicas, fisiológicas e morfológicas que indicam a exposição ou o efeito tóxico de um determinado poluente em um organismo-teste.

No Brasil, vários estudos utilizam biomarcadores de contaminação aquática e demonstraram a utilidade dessa metodologia e o alto grau de impacto ambiental nas regiões costeiras

Recebido em 27 de agosto de 2011

Aceito em 1 de outubro de 2012

E-mail: deborabpsousa@hotmail.com 
(Au, 2004; Belotto et al., 2009; Pereira et al., 2011). Nas regiões Norte e Nordeste do Brasil, estudos dessa abrangência ainda são restritos e indicam a necessidade de biomonitoramento que estabeleça os potenciais impactos sofridos por espécies nativas em regiões estuarinas (Carvalho-Neta e Abreu-Silva, 2010; Montes et al., 2010).

Estudos que esclareçam o potencial e a dinâmica dos xenobióticos ou de outros contaminantes nas populações ícticas de importância econômica para o estado do Maranhão são de grande relevância, uma vez que a pesca artesanal ainda é realizada próximo ao complexo portuário de São Luís, MA, onde esses organismos podem estar sujeitos a condições fisiológicas desestabilizadoras em função dos contaminantes oriundos da carga e descarga de navios. Entre as espécies de peixes que mais sofrem os efeitos desses contaminantes, destacam-se as bentônicas, como, por exemplo, os bagres Sciades herzbergii e Bagre bagre, que apresentam grande importância nas pescarias artesanais de várias regiões da costa brasileira.
Assim, no presente trabalho, objetivou-se validar as lesões branquiais como biomarcadores para a avaliação dos efeitos de contaminantes ambientais nas populações de Sciades herzbergii e Bagre bagre da Baía de São Marcos, MA, a fim de selecionar um táxon como bioindicador capaz de ser utilizado em programas de biomonitoramento na região.

\section{MATERIAL E MÉTODOS}

Espécimes de $S$. herzbergii e $B$. bagre foram amostrados em dois locais distintos na Baía de São Marcos (Fig. 1) no período de abril a outubro de 2010. A Ilha dos Caranguejos (S1), localizada sob as coordenadas 02 $49^{\prime} 06^{\prime}$ 'S e $044^{\circ} 29^{\prime} 05^{\prime}$ ' $\mathrm{W}$, foi utilizada como área de referência por ser uma Unidade de Conservação, criada pelo governo do estado do Maranhão. A região de influência do complexo portuário (S2) de São Luís, MA, próximo ao porto da ALUMAR/ALCOA, 02\%43'14',S e $044^{\circ} 23^{\prime} 35^{\prime}$ 'W, foi considerada uma área potencialmente contaminada.

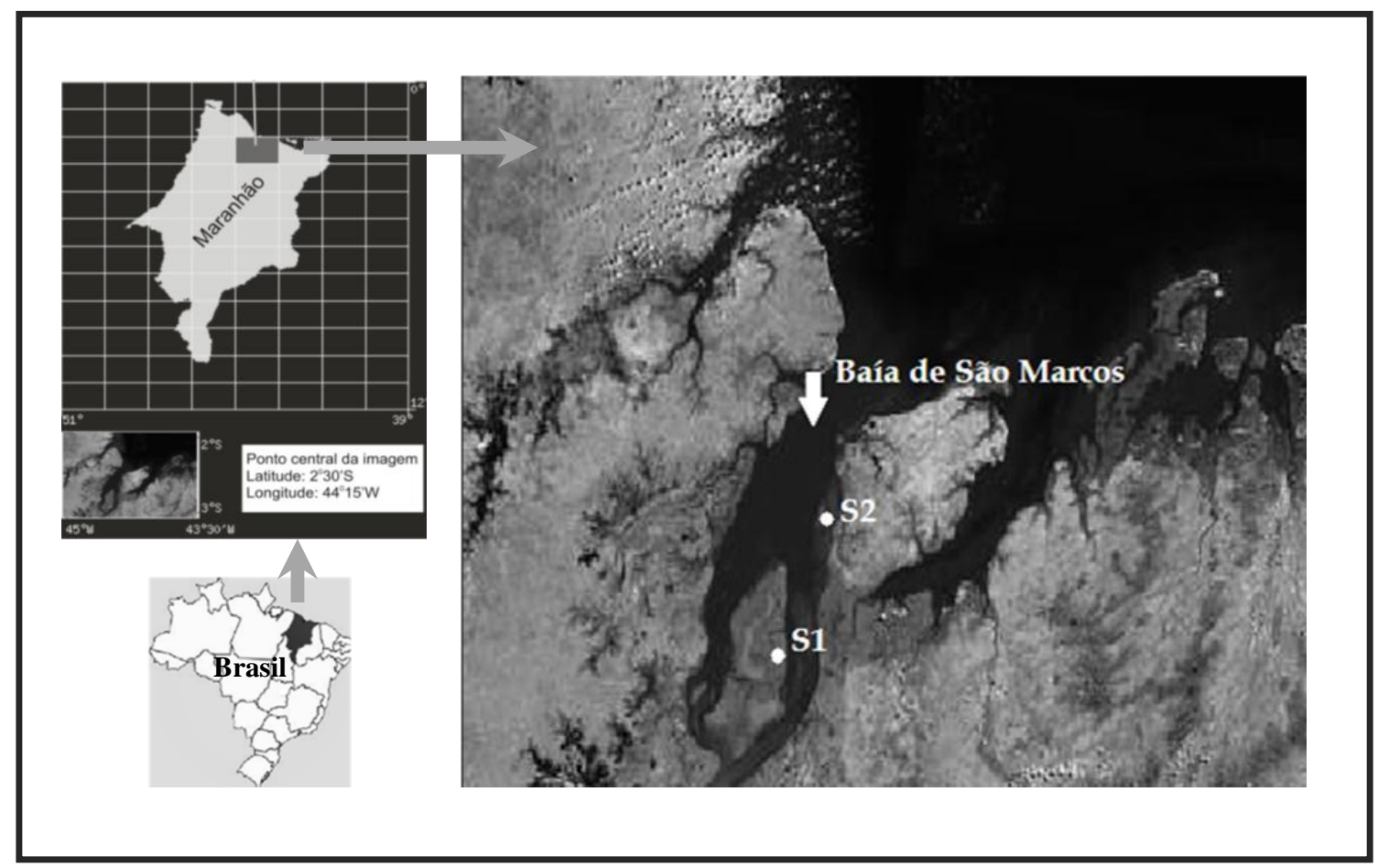

Figura 1. Pontos de amostragem de Sciades herzbergii e Bagre bagre na Baía e São Marcos, Maranhão. Fonte: Zoneamento Ecológico-Econômico do Estado do Maranhão (2011). 
Nos locais de coleta foram aferidos os dados de salinidade, temperatura, $\mathrm{pH}$ e oxigênio dissolvido em cada área sujeita a investigação. Dos exemplares de $S$. herzbergii e $B$. bagre capturados foram registrados dados biométricos de comprimento total (LT), comprimento padrão (LP), comprimento furcal (LF), peso total (WT) e o peso das gônadas (WG). Também foi calculado o índice gonadossomático (GSI), conforme Vazoller (1996): GSI = Wg.100/Wt.

Em laboratório, 120 brânquias foram fixadas em formol a $10 \%$ e mantidas em álcool a $70 \%$ até o processamento histológico usual. Para tanto, foram aplicadas ao primeiro arco branquial direito técnicas histológicas usuais e inclusão em parafina. Cortes transversais, de aproximadamente $5 \mu \mathrm{m}$ de espessura, foram corados com hematoxilina e eosina (HE). A leitura das lâminas foi realizada em microscópio óptico e as lesões foram posteriormente fotomicrografadas em fotomicroscópio AXIOSKOP - ZEIS. Os resultados dos dados biométricos dos peixes foram expressos como média \pm desvio padrão para machos e fêmeas, sendo comparados por meio do teste-t. O nível de significância considerado foi de 0,05 .

\section{RESULTADOS}

Os valores médios das variáveis abióticas registradas na Baía de São Marcos durante as quatro coletas foram agrupados em "período de estiagem" e "período chuvoso" (Tab. 1). A salinidade mostrou-se uniforme nas duas áreas amostradas, decrescendo no período das chuvas em ambas as áreas. $\mathrm{O}$ oxigênio dissolvido e a saturação de oxigênio dissolvido foram sempre menores na área potencialmente contaminada. Os valores de $\mathrm{pH}$ e turbidez mantiveram-se constantes para as duas áreas.

Tabela 1. Parâmetros ambientais analisados nos seis pontos de coleta de cada região da Baía de São Marcos, Maranhão, no período de estiagem (abril/2010) e na época chuvosa (outubro/2010)

\begin{tabular}{|c|c|c|c|c|c|c|c|c|c|c|c|c|}
\hline \multirow{3}{*}{ Parâmetro } & \multicolumn{6}{|c|}{ S2 (potencialmente contaminado) } & \multicolumn{6}{|c|}{ S1 (referência) } \\
\hline & \multicolumn{3}{|c|}{ Período de estiagem } & \multicolumn{3}{|c|}{ Período chuvoso } & \multicolumn{3}{|c|}{ Período de estiagem } & \multicolumn{3}{|c|}{ Período chuvoso } \\
\hline & $\begin{array}{c}\text { Ponto } \\
1\end{array}$ & $\begin{array}{c}\text { Ponto } \\
2\end{array}$ & $\begin{array}{c}\text { Ponto } \\
3\end{array}$ & $\begin{array}{c}\text { Ponto } \\
1\end{array}$ & $\begin{array}{c}\text { Ponto } \\
2\end{array}$ & $\begin{array}{c}\text { Ponto } \\
3\end{array}$ & $\begin{array}{c}\text { Ponto } \\
1\end{array}$ & $\begin{array}{c}\text { Ponto } \\
2\end{array}$ & $\begin{array}{c}\text { Ponto } \\
3\end{array}$ & $\begin{array}{c}\text { Ponto } \\
1\end{array}$ & $\begin{array}{c}\text { Ponto } \\
2\end{array}$ & $\begin{array}{c}\text { Ponto } \\
3\end{array}$ \\
\hline Temp. $\left({ }^{\circ} \mathrm{C}\right)$ & 29,1 & 29,1 & 29,1 & 29,0 & 29,0 & 29,0 & 29,0 & 29,0 & 29,0 & 29,0 & 29,0 & 29,0 \\
\hline Salin. (UPS) & 15 & 15 & 15 & 14 & 14 & 14 & 15 & 15 & 15 & 10 & 10 & 10 \\
\hline $\mathrm{Ph}$ & 8,2 & 8,1 & 8,3 & 8,2 & 8,1 & 8,3 & 8,0 & 8,1 & 8,1 & 8,1 & 8,1 & 8,1 \\
\hline $\mathrm{OD}\left(\mathrm{mg} / \mathrm{L} \mathrm{O}_{2}\right)$ & 4,9 & 4,9 & 4,9 & 5,1 & 5,1 & 5,1 & 6,0 & 6,0 & 5,9 & 6,1 & 6,1 & 6,1 \\
\hline$\%$ satur. OD & 86,2 & 86,3 & 86,1 & 86,3 & 86,7 & 86,4 & 88,6 & 88,7 & 88,6 & 88,9 & 88,8 & 88,7 \\
\hline Turbidez(NTU) & 12,0 & 12,0 & 13,0 & 14,0 & 13,0 & 13,0 & 13,0 & 13,0 & 13,0 & 13,0 & 13,0 & 13,0 \\
\hline
\end{tabular}

Os dados biométricos para machos e fêmeas de S. herzbergii são apresentados na Tab. 2, e indicaram que o comprimento total e furcal dos peixes capturados no local potencialmente contaminado foi menor $(\mathrm{P}<0,05)$ do que no local de referência.

O percentual de estágios gonadais dos peixes está indicado na Tab. 3. Os dados mostram peixes da área de referência em todos os estágios gonadais, principalmente no EG4, indicando atividade reprodutiva das fêmeas. No local potencialmente contaminado, não foram encontrados peixes juvenis (EG1).
Os dados biométricos para machos e fêmeas de Bagre bagre são apresentados na Tab. 4, e indicaram o mesmo padrão do táxon anterior, com comprimento total e furcal dos peixes capturados no local potencialmente contaminado (S2) menor $(\mathrm{P}<0.05)$ do que no local de referência $(\mathrm{S} 1)$.

Os percentuais de estágios gonadais dos peixes estão indicados na Tabela 5. De forma geral, constatou-se para fêmeas e machos predominância de indivíduos em maturação (EG2) na área potencialmente contaminada. 
Tabela 2. Dados biométricos de machos e fêmeas de $S$. herzbergii coletados na área potencialmente contaminada (S2) e de referência (S1) da Baía de São Marcos

\begin{tabular}{|c|c|c|c|c|c|}
\hline \multirow{3}{*}{ Parâmetro } & \multicolumn{4}{|c|}{ média \pm desvio-padrão } & \multirow[b]{3}{*}{ Fêmea / Macho } \\
\hline & \multicolumn{2}{|c|}{ S2 (potencialmente contaminado) } & \multicolumn{2}{|c|}{ S1 (referência) } & \\
\hline & Fêmea & Macho & Fêmea & Macho & \\
\hline LT & $25,16 \pm 4,5(\mathrm{~cm})$ & $28,28 \pm 3,64(\mathrm{~cm})$ & $39,49 \pm 1,4(\mathrm{~cm})$ & $39,48 \pm 1,64(\mathrm{~cm})$ & $0,0013 * / 0,001$ \\
\hline $\mathrm{LF}$ & $21,2 \pm 4,5(\mathrm{~cm})$ & $24,26 \pm 3,6(\mathrm{~cm})$ & $35,5 \pm 1,70(\mathrm{~cm})$ & $36,50 \pm 1,60(\mathrm{~cm})$ & $0,062 * / 0,001$ \\
\hline WT & $186,2 \pm 45,1(\mathrm{~g})$ & $123,34 \pm 58,35(\mathrm{~g})$ & $296,4 \pm 18,1(\mathrm{~g})$ & $291,7 \pm 15,19(\mathrm{~g})$ & $0,001 / 0,017$ \\
\hline Wg & $1,01 \pm 0,84(\mathrm{~g})$ & $1,09 \pm 0,95(\mathrm{~g})$ & $1,75 \pm 0,19(\mathrm{~g})$ & $1,58 \pm 0,16(\mathrm{~g})$ & $0,242 / 0,956$ \\
\hline GSI & $0,87 \pm 0,38$ & $0,86 \pm 0,35$ & $3,66 \pm 0,06$ & $11,80 \pm 0,35$ & $0,014 / 0,022$ \\
\hline
\end{tabular}

*Indica diferença estatística em relação à área de referência $(\mathrm{P}<0.05)$. Número de indivíduos $=60$. Número de fêmeas: $\mathrm{S} 1=15$; $\mathrm{S} 2=15$. Número de machos: $\mathrm{S} 1=15$; $\mathrm{S} 2=15$. LT: comprimento total; LF: comprimento furcal; WT: peso total; WG: peso das gônadas; GSI: índice gonadossomático = WG.100/WT.

Tabela 3. Estágios de maturação gonadal de machos e fêmeas de S. herzbergii coletados na Baía de São Marcos

\begin{tabular}{ccccc}
\hline EG & \multicolumn{2}{c}{ S2 (potencialmente contaminado) } & \multicolumn{2}{c}{ S1 (referência) } \\
\cline { 2 - 5 } & Fêmea & Macho & Fêmea & Macho \\
\hline EG1 & $0 \%$ & $0 \%$ & $16 \%$ & $22 \%$ \\
EG2 & $55 \%$ & $60 \%$ & $14 \%$ & $13 \%$ \\
EG3 & $6 \%$ & $7 \%$ & $24 \%$ & $32 \%$ \\
EG4 & $22 \%$ & $25 \%$ & $54 \%$ & $29 \%$ \\
\hline
\end{tabular}

EG: estágio gonadal.

Tabela 4. Dados biométricos de machos e fêmeas de B. bagre coletados na área potencialmente contaminada (S2) e de referência (S1) da Baía de São Marcos

\begin{tabular}{|c|c|c|c|c|c|}
\hline \multirow{3}{*}{ Parâmetro } & \multicolumn{4}{|c|}{ Média \pm desvio-padrão } & \multirow{3}{*}{$\begin{array}{c}\text { Fêmea/Mach } \\
\text { o }\end{array}$} \\
\hline & \multicolumn{2}{|c|}{ S2 (potencialmente contaminado) } & \multicolumn{2}{|c|}{ S1 (referência) } & \\
\hline & Fêmea & Macho & Fêmea & Macho & \\
\hline LT & $27,92 \pm 3,22(\mathrm{~cm})$ & $25,21 \pm 3,87(\mathrm{~cm})$ & $35,08 \pm 1,98(\mathrm{~cm})$ & $28,37 \pm 3,78(\mathrm{~cm})$ & $0,04 * / 0,28$ \\
\hline LF & $22,93 \pm 2,71(\mathrm{~cm})$ & $20,96 \pm 3,09(\mathrm{~cm})$ & $29,0 \pm 1,58(\mathrm{~cm})$ & $23,18 \pm 2,90(\mathrm{~cm})$ & $0,01 * / 0,001$ \\
\hline WT & $136,25 \pm 27,46(\mathrm{~g})$ & $100,32 \pm 37,91(\mathrm{~g})$ & $288,33 \pm 47,50(\mathrm{~g})$ & $154,16 \pm 61,0(\mathrm{~g})$ & $0,01 / 0,15$ \\
\hline $\mathrm{Wg}$ & $8,76 \pm 8,97(\mathrm{~g})$ & $0,78 \pm 2,16(\mathrm{~g})$ & $10,88 \pm 9,32(\mathrm{~g})$ & $2,60 \pm 3,06(\mathrm{~g})$ & $0,35 / 0,11$ \\
\hline GSI & $3,26 \pm 3,10$ & $0,24 \pm 0,20$ & $4,31 \pm 3,68$ & $2,87 \pm 2,15$ & $0,90 / 0,14$ \\
\hline
\end{tabular}

*Indica diferença estatística em relação à área de referência $(\mathrm{P}<0.05)$. Número de indivíduos $=60$. Número de fêmeas: $\mathrm{S} 1=7 ; \mathrm{S} 2=30$. Número de machos: $\mathrm{S} 1=23 ; \mathrm{S} 2=15$. LT: comprimento total; LF: comprimento furcal; WT: peso total; WG: peso das gônadas; GSI: índice gonadossomático = WG.100/WT.

Tabela 5. Estágio de maturação gonadal de machos e fêmeas de B. bagre coletados na Baía de São Marcos

\begin{tabular}{ccccc}
\hline \multirow{2}{*}{ EG } & \multicolumn{2}{c}{ S2 (potencialmente contaminado) } & \multicolumn{2}{c}{ S1 (referência) } \\
\cline { 2 - 5 } & Fêmea & Macho & Fêmea & Macho \\
\hline EG1 & $12 \%$ & $15 \%$ & $26 \%$ & $22 \%$ \\
EG2 & $30 \%$ & $22 \%$ & $30 \%$ & $35 \%$ \\
EG3 & $10 \%$ & $15 \%$ & $35 \%$ & $28 \%$ \\
EG4 & $5 \%$ & $6 \%$ & $25 \%$ & $18 \%$ \\
\hline
\end{tabular}

EG: estágio gonadal.

A análise histopatológica realizada nos exemplares de $S$. herzbergii da Ilha dos Caranguejos não mostrou alterações morfológicas nas brânquias dos bagres. Os indivíduos coletados na área impactada, sob influência do complexo portuário, apresentaram várias alterações histológicas. Os principais tipos de alterações histopatológicas encontradas foram estreitamento lamelar e teleangectasia (Fig. 2). A análise das brânquias dos exemplares de $B$. bagre 
indicou alterações morfológicas do tipo estreitamento lamelar e teleangectasia em indivíduos tanto da área de referência (S1) quanto da área potencialmente contaminada (S2). As principais alterações encontradas foram: estreitamento lamelar, teleangectasia, fusão das lamelas secundárias e descolamento do epitélio das lamelas secundárias (Fig. 3).

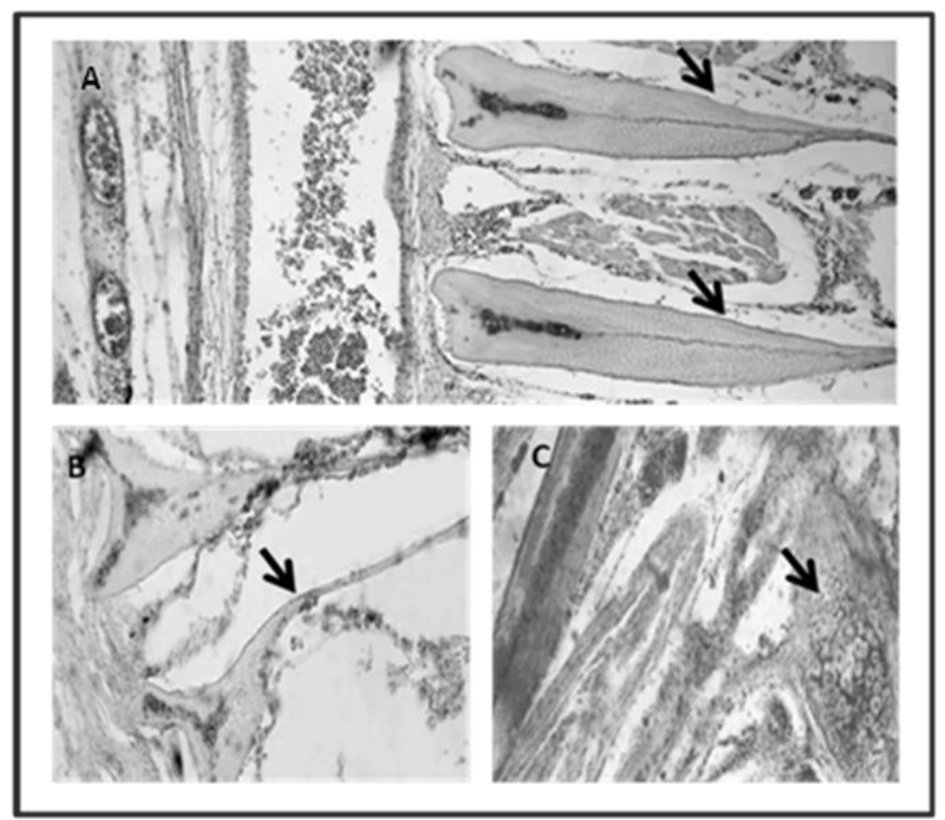

Figura 2. Fotomicrografia do primeiro arco branquial direito de exemplar de Sciades herzbergii da Baía de São Marcos. Detalhe de filamentos branquiais mostrando duas lamelas primárias (A) sem alterações histológicas (setas); (B) estreitamento lamelar (seta); (C) teleangectasia. X $100 \mathrm{HE}$

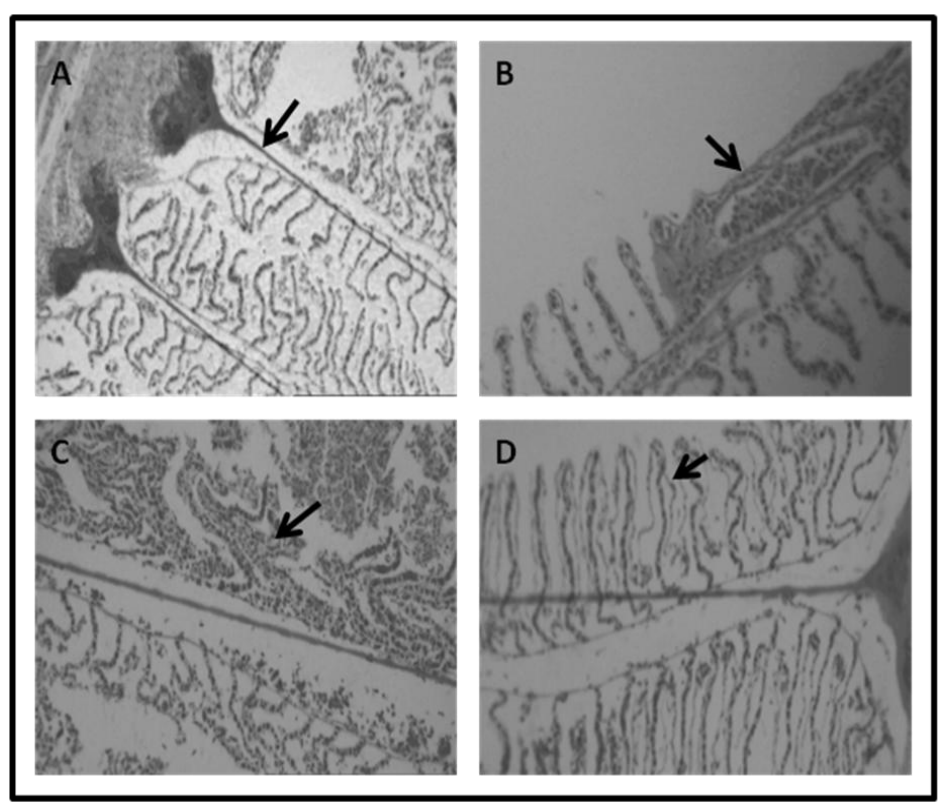

Figura 3. Fotomicrografia do primeiro arco branquial direito de exemplar de Bagre bagre da Baía de São Marcos. Detalhe de filamentos branquiais mostrando duas lamelas primárias (A) estreitamento lamelar (setas); (B) teleangectasia (seta); (C) fusão das lamelas secundárias (seta); (D) descolamento do epitélio das lamelas secundárias (seta). X 100 HE. 


\section{DISCUSSÃO}

As duas áreas da Baía de São Marcos analisadas neste trabalho apresentaram fatores abióticos ( $\mathrm{pH}$, temperatura, salinidade e turbidez) considerados normais de acordo com outros trabalhos realizados para a região (CarvalhoNeta et al., 2012; Sousa e Carvalho-Neta, 2011). Segundo Siqueira et al. (2003), esse padrão é característico das regiões tropicais onde as temperaturas são uniformes e apresentam poucas flutuações ao longo do ano.

Os dados biométricos dos exemplares de $S$. herzbergii mostraram diferenças significativas entre a área de referência (S1) e o local potencialmente contaminado (S2). Todos os valores dos dados biométricos e índice gonadossomático de machos e fêmeas de S1 foram superiores aos dos indivíduos de S2. Esses resultados indicam maior atividade reprodutiva na área de referência, pois valores de GSI mais elevados expressam amadurecimento adequado das gônadas (Vazzoler, 1996). Nicholson e Lam (2005) afirmaram que a presença de xenobiontes em ambientes aquáticos leva à perda de funcionalidade de várias reservas energéticas dos animais (em especial no processo reprodutivo) como um mecanismo compensatório à alta demanda de energia requerida pelos processos de detoxificação.

As brânquias de $S$. herzbergii não apresentaram alterações histológicas apenas para indivíduos amostrados na área de referência. De acordo com Klontz (1972), peixes são intimamente associados com seu ambiente aquoso, e as mudanças físicas e químicas do ecossistema são rapidamente refletidas como medidas fisiológicas quantificáveis nos peixes. Esse diagnóstico de lesões branquiais demonstra os possíveis graus de estresse a que os organismos amostrados estavam submetidos.

As regiões portuárias brasileiras são consideradas fontes pontuais de substâncias tóxicas, cujos poluentes podem afetar os ecossistemas marinhos via transporte de sedimento e difusão dessas substâncias nos corpos aquáticos. Sendo assim, é de extrema importância o monitoramento desses ambientes (Oliveira e Madureira, 2009) com a utilização de biomarcadores em populações de organismos que respondem aos impactos nessas localidades.
O exame histopatológico do epitélio branquial dos bagres permitiu diferenciar claramente a região da Ilha dos Caranguejos e do complexo portuário da Baía de São Marcos. A elevada incidência de alterações severas indica que os peixes da região portuária estão apresentando respostas biológicas quantificáveis ao estresse local.

A informação necessária para predizer os efeitos de distúrbios ambientais inclui não somente quais organismos estão presentes e como eles variam no tempo e no espaço, mas também quais fatores controlam essa variação e como eles provavelmente responderam ao distúrbio (Morrissey, 1993). Na área analisada, onde foram utilizados dados de GSI e lesões branquiais, verificou-se a importância de se analisar esses parâmetros de forma integrada, uma vez que foi observada uma provável alteração reprodutiva (inexistência de juvenis) associada a lesões branquiais nos adultos apenas na área potencialmente contaminada para $S$. herzbergii.

Em B. bagre verificou-se a presença de várias alterações morfológicas em indivíduos tanto da área de referência quanto da área potencialmente contaminada. Essas mesmas histopatologias em brânquias foram encontradas também em estudos realizados por Guarcia-Santos et al. (2007) com tilápias do Nilo (Oreochromis niloticus) quando expostas ao cádmio, e por Santos et al. (2012) com a mesma espécie e com o híbrido tambacu (Colossoma macropomum fêmea e Piaractus mesopotamicus macho).

A sensibilidade da ictiofauna aos poluentes pode variar de acordo com a suscetibilidade da espécie, a sazonalidade e sua capacidade de migração (Bernet et al., 1999). A capacidade de migração e a grande mobilidade de $B$. bagre parece ser o processo que melhor explica a existência das lesões branquiais observadas nos indivíduos de ambas as áreas amostradas. De acordo com Carvalho-Neta e Castro (2008), na Ilha dos Caranguejos, B. bagre é uma espécie migrante-marinha que ocorre com baixa regularidade ao longo do ano nos igarapés da região. Indivíduos dessa espécie visitam muitas áreas ao longo da costa Norte do Brasil, sendo possível a sua contaminação em áreas distantes da Ilha dos Caranguejos. 
Foi possível identificar apenas $S$. herzbergii como uma espécie bioindicadora, cujas respostas biológicas podem ser utilizadas para comparar uma área impactada e uma área de referência. Os bioindicadores são organismos, grupos de espécies ou comunidades biológicas cuja presença, abundância e condições são indicativos biológicos de uma determinada condição ambiental, sendo importantes para avaliar um determinado fator antrópico ou um fator natural com potencial impactante (Callisto et al., 2005).

A espécie estuarina-residente (S. herzbergii) mostrou-se mais apropriada para a análise de biomarcadores de contaminação aquática porque os indivíduos estão estabelecidos ecologicamente tanto em áreas protegidas quanto em áreas impactadas. Suas respostas biológicas alteradas foram registradas apenas no complexo portuário, em cujas águas e sedimento foram detectados vários poluentes acima dos níveis permitidos pela legislação ambiental brasileira (Carvalho Neta et al., 2012). Já a espécie migrante marinha (B. bagre) não possibilitou essa diferenciação, visto que mostrou alterações branquiais nas duas áreas, provavelmente em função dos processos migratórios e fisiológicos dos indivíduos.

Estudos de biomonitoramento realizados por Montes et al. (2010) na Baía do Guajará, no estado do Pará, mostraram que o exame histopatológico realizado em brânquias de Brachyplatystoma rousseauxii foi considerado um excelente biomarcador e que essa espécie nativa pode ser utilizada como bioindicadora de ambientes impactados ou protegidos daquela região. Esses dados reforçam a importância do uso de diferentes metodologias de biomonitoramento dos ecossistemas estuarinos na costa Norte do Brasil, enfatizando a utilização de lesões branquiais em espécies nativas como biomarcadores de contaminação aquática capazes de auxiliar na avaliação mais precisa da qualidade ambiental com relativo baixo custo e rapidez.

O monitoramento contínuo, através da utilização desse tipo de biomarcadores em peixes, associado aos parâmetros ambientais e químicos, nas áreas de influência portuária, poderá oferecer um diagnóstico seguro sobre a saúde das comunidades ícticas e dos ecossistemas aquáticos.

\section{CONCLUSÕES}

O táxon Sciades herzbergii mostrou ser um bioindicador apropriado para avaliar a qualidade ambiental da Unidade de Conservação (Ilha dos Caranguejos) e indicar impacto ambiental na área de influência do complexo portuário instalado na Baía de São Marcos, sendo as lesões branquiais validadas como biomarcadores de contaminação aquática nessa espécie estuarino-residente. $\mathrm{Na}$ área de referência (Ilha dos Caranguejos), foram encontradas alterações histopatológicas nas brânquias de Bagre bagre, provavelmente oriundas da exposição desses peixes aos poluentes de outras regiões visitadas por esses organismos migrantes.

\section{AGRADECIMENTOS}

Os nossos agradecimentos à FAPEMA e ao $\mathrm{CNPq}$ pelo suporte financeiro. À equipe do LabPEA (Laboratório de Pesca e Ecologia Aquática) pelo apoio durante as coletas e análises dos peixes. Ao pesquisador e biólogo Hilberto Silva Cutrim pela colaboração durante o processamento histológico e confecção das lâminas.

\section{REFERÊNCIAS}

AU, D. The application of histo-cytopathological biomarkers in marine monitoring: A review. Mar. Pollut. Bull., v.48, p.817-834, 2004.

BELOTTO, R.V.; BRITO, P.C.; MARTINS, L.K.P. et al. Desestabilização da membrana lisossômica em hemócitos de Mytella guyanensis (Lamarck, 1819) (MOLUSCA-BIVALVIA) como biomarcador de estresse ambiental nos Manguezais da baía de Babitonga (Santa Catarina, Brasil). Rev. Gest. Cost. Integra., v.2, p.1-7, 2009.

BERNET, D.; SCHIMIDT, H.; MEIER, W. et al. Histophatology in Fish: Proposal for a Protocol to Assess Aquatic Polluition. J. Fis. Disea., v.22, p.25-34, 1999.

CALlisto, M.; GONÇALVES, J.F.; MORENO, P. Invertebrados Aquáticos como bioindicadores. In: GOULART, E.M.A. (Ed.). Navegando o Rio das Velhas das Minas aos Gerais. Belo Horizonte: UFMG, 2005. p.555-567. 
CARVALHO-NETA, R.N.F.; ABREU-SILVA, A.L. Sciades herzbergii oxidative stress biomarkers: An in situ study of estuarine ecosystem (São Marcos’ Bay, Maranhão, Brazil). Braz. J. Ocean., v.58, p.11-17, 2010.

CARVALHO-NETA, R.N.F.; CASTRO, A.C.L. Diversidade das assembléias de peixes estuarinas na Ilha dos Caranguejos, Maranhão. Arq. Cienc. Mar, n.41, p.48-57, 2008.

CARVALHO-NETA, R.N.F.; TORRES JR., A.R.; ABREU-SILVA, A.L. Biomarkers in Catfish Sciades herzbergii (Teleostei: Ariidae) from Polluted and Non-polluted Areas (São Marcos' Bay, Northeastern Brazil). Appl. Bioch. Biot., v.166, p.1-12, 2012.

FERNANDES, A.F. The use of biomarks in aquatic toxicology studies. Rev. Port. Zootec., v.12, p.67-86, 2005.

GUARCIA-SANTOS, S.; MONTEIRO, S.M.; CARROLA, J. et al. Alterações histológicas em brânquias de tilápia nilotica Oreochromis niloticus causadas pelo cádmio. Arq. Bras. Med. Vet. Zootec., v.59, p.376-381, 2007.

HUGGETT, R.J.; KIMERIE, P.M.; BERGMAN, H.L. (Ed). Biomarks: Biochemical, Physiolocal and Histological markers of anthropogenic stress. Boca Raton: Lewis Publishers, 1992. 347p.

KLONTZ, G.W. Hematological techiques and the immune response in rainbow trout. Symp. Zool. Soc. Lond., v.30, p.89-99, 1972.

MORRISSEY, D.J. Environmental Impact Assessment - A Review of its Aims end Recent Developments. Mar. Pollut. Bullet., v.26, p.540$545,1993$.

MONTES, C.S.; FERREIRA, M.A.P.; SANTOS, S.S.D. et al. Branchial histopathological study of Brachyplatystoma rousseauxii (Castelnau, 1855) in the Guajará bay, Belém, Pará State, Brazil. Act. Scient. Biolog. Sci., v.32, p.93-99, 2010.

NICHOLSON, S.; LAM, P.K.S. Pollution monitoring in Southest Asia using biomarkers in the mytilid mussel Perna viridis (Mytilidae: Bivalvia). Environ. Int., v.31, p.121-132, 2005.
OLIVEIRA， C.R.; MADUREIRA， L.A.S. Distribuição de hopanos e avaliação da contaminação por derivados de petróleo em sedimentos superficiais na costa portuária de Santa Catarina, Brasil. In: $32^{\circ}$ REUNIÃO ANUAL DA SOCIEDADE BRASILEIRA DE QUÍMICA, 32, 2009, Fortaleza. Anais... Fortaleza: [s.n.] 2009. p.97. (Resumo).

PEREIRA， C.D.; MARTIN-DÍAZ, M.L.; ZANETTE, J.C.A. et al. Integrated biomarker responses as environmental status descriptors of a coastal zone (São Paulo, Brazil). Ecotox. Environ. Safe., v.74, p.1257-1264, 2011.

SANTOS, D.M.S.; CRUZ, C.F.; PEREIRA, D.P. et al. Microbiological water quality and gill histopathology of fish from fish farming in Itapecuru-Mirim County, Maranhão State. Act. Sci. Biol. Sci., v.34, p.199-205, 2012.

SIQUEIRA, G.W.; LIMA, W.N.; MENDES, A.S. et al. Aspectos hidroquímicos das águas subjacentes à Plataforma Continental do Amazonas entre os Cabos Orange (AP) e Maguari (PA). Bol. Lab. Hidrob., v.16, p.5-20, 2003.

SOUSA, D.B.P.; CARVALHO-NETA, R.N.F. Lesões branquiais como biomarcadores de contaminação aquática em Sciades herzbergii (Siluriformes, Ariidae) da Baía de São Marcos, Maranhão. In: CONGRESSO BRASILEIRO DE BIOLOGIA MARINHA (CBBM), 3., 2011, Natal. Anais... Natal: [s.n.] 2011. (Resumo).

VAZZOLER, A.E.M. (Ed). Biologia $e$ reprodução de peixes teleósteos: teoria e prática. Maringá: EDUEM, 1996. 169p.

ZONEAMENTO ECOLÓGICO-ECONÔMICO DO ESTADO DO MARANHÃO. Mapas temáticos. São Luís. Disponível em: <http://www.zee.ma.gov.br/>. Acessado em: 10 ago. 2011 . 